\title{
A importância da monitoria remota na formação acadêmica do estudante de Enfermagem
}

The importance of remote monitoring in the academic education of nursing students

La importancia del supervision remoto en la formación académica de estudiante de enfermeira

Franciare Vieira Silva

ORCID: https://orcid.org/0000-0001-5914-6344 Faculdade CESMAC do Sertão, Brasil E-mail: franciare123@hotmail.com

João Paulo do Nascimento Cordeiro ORCID: https://orcid.org/0000-0003-3407-1328 Faculdade CESMAC do Sertão, Brasil E-mail: joaopaulodnc@gmail.com

Lissa Lavínia de Oliveira Silva ORCID: https://orcid.org/0000-0003-3626-0181 Faculdade CESMAC do Sertão, Brasil E-mail: laviniao771@gmail.com

Anny Stphane Temoteo Pereira ORCID: https://orcid.org/0000-0003-1663-3753 Faculdade CESMAC do Sertão, Brasil E-mail: annystphanett@gmail.com

Giovanna Canuto Duarte

ORCID: https://orcid.org/0000-0002-1672-9942 Faculdade CESMAC do Sertão, Brasil E-mail: duartegiovanna123@gmail.com

Emmaely Alves da Silva

ORCID: https://orcid.org/0000-0001-7022-0103 Faculdade CESMAC do Sertão, Brasil E-mail: emmaelyalves6@gmail.com

Lísia Marília Tenório da Silva ORCID: https://orcid.org/0000-0002-1585-7627 Faculdade CESMAC do Sertão, Brasil

E-mail: lisiamarilia@hotmail.com

Emanuelly Kesley de Freitas Lima ORCID: https://orcid.org/0000-0002-8456-5775 Faculdade CESMAC do Sertão, Brasil E-mail: manufr11999@hotmail.com

Carla Souza dos Anjos ORCID: https://orcid.org/0000-0002-8403-9943 Universidade Federal de Alagoas, Brasil E-mail: carla.anjos@arapiraca.ufal.br Eliane Duarte Araújo

ORCID: https://orcid.org/0000-0002-3672-3142 Faculdade Sequencial, Brasil E-mail: elianeanjo@live.com

Júlia Pereira de Moura Ferreira ORCID: https://orcid.org/0000-0002-4228-4318 Faculdade CESMAC do Sertão, Brasil E-mail: juliap201416@gmail.com

Andressa Almeida Santos

ORCID: https://orcid.org/0000-0002-9299-8247 Faculdade CESMAC do Sertão, Brasil

E-mail: andressaalmeida2000aa@gmail.com Maria Victoria Guimarães Ramos Valeriano Cavalcante ORCID: https://orcid.org/0000-0001-9677-135X Faculdade CESMAC do Sertão, Brasil E-mail: Mvgrvc00@gmail.com

Thaís da Silva Alves de Lemos ORCID: https://orcid.org/0000-0002-1995-2779 Faculdade CESMAC do Sertão, Brasil E-mail: thaisinhachagas30@gmail.com 


\author{
Marcela Farias dos Santos \\ ORCID: https://orcid.org/0000-0003-1580-2631 \\ Faculdade CESMAC do Sertão, Brasil \\ E-mail: marcelafariassan@outlook.com \\ Mayra Bianca Lopes Lima \\ ORCID: https://orcid.org/0000-0002-8167-0799 \\ Faculdade CESMAC do Sertão, Brasil \\ E-mail: mayrabianca42@hotmail.com \\ Laura Beatriz Lima dos Santos \\ ORCID: https://orcid.org/0000-0003-2342-8344 \\ Faculdade CESMAC do Sertão, Brasil \\ E-mail: laurabiaa13@gmail.com \\ Rafaella dos Anjos Bomfim \\ ORCID: https://orcid.org/0000-0003-3474-4967 \\ Faculdade CESMAC do Sertão, Brasil \\ E-mail: rafaellabomfim@hotmail.com \\ Núbia Ivo da Silva \\ ORCID: https://orcid.org/0000-0002-7857-9185 \\ Faculdade CESMAC do Sertão, Brasil \\ E-mail:Nubbiaivo@gmail.com \\ Daniele Cristina de Oliveira Lima \\ ORCID: https://orcid.org/0000-0003-4248-697X \\ Docente da Faculdade CESMAC do Sertão, Brasil \\ E-mail: daniele.silva@cesmac.edu.br
}

\begin{abstract}
Resumo
A monitoria é uma das atividades de ensino, pesquisa e extensão mais importantes das instituições de nível superior, havendo também a monitoria remota, que conjectura o mesmo objetivo e diretrizes da monitoria presencial. Nesse sentido, o artigo tem como objetivo descrever a experiência vivenciada por acadêmicas do curso de graduação em enfermagem da Faculdade CESMAC do Sertão. Trata-se de um estudo descritivo, do tipo relato de experiência, cuja narrativa se baseia em uma observação sistemática da realidade. Foi elaborado um relato de experiência, com o intuito de demonstrar ferramentas inovadoras para o ensino-aprendizagem, descrevendo as Tecnologias de Comunicação e Informação utilizadas. Nessa perspectiva, diante do cenário da COVID-19 novas medidas tiveram que ser adotadas, e, assim, a faculdade adotou o projeto de monitoria remota que foi distribuída em disciplinas de determinados períodos, possibilitando experiências, promovendo a integração entre os alunos, apoio ao desenvolvimento da disciplina e no esclarecimento de dúvidas. Desse modo, o programa de monitoria remota foi ministrado pelos discentes da faculdade sob a orientação dos respectivos professores das disciplinas. A partir deste estudo, nota-se a importância da monitoria remota para os alunos, de forma que essa modalidade de ensino-aprendizagem contribuiu na formação de estudantes, beneficiando tanto aquele na função de monitor como aquele que se encontra enquanto ouvinte/parceiro de aprendizado, compartilhando conhecimentos, agregando a si uma nova forma de enxergar assuntos e práticas, e, portanto, sendo fundamental em momentos futuros, nos quais o discente fará de si um melhor profissional.
\end{abstract}

Palavras-chave: Ensino; Aprendizagem; Saúde; Enfermagem; Covid-19.

\begin{abstract}
Monitoring is one of the most important teaching, research and extension activities of higher education institutions, and there is also remote monitoring, which has the same objective and guidelines as in-person monitoring. In this sense, the article aims to describe the experience lived by students from the undergraduate nursing course at the CESMAC School of Sertão. This is a descriptive study, of the experience report type, whose narrative is based on a systematic observation of reality. An experience report was prepared in order to demonstrate innovative tools for teaching-learning, describing the Communication and Information Technologies used. In this perspective, facing the COVID-19 scenario new measures had to be adopted, and, thus, the college adopted the remote monitoring project that was distributed in disciplines of certain periods, enabling experiences, promoting the integration among students, supporting the development of the discipline and clarifying doubts. Thus, the remote monitoring program was taught by the college's students under the guidance of their respective subject teachers. From this study, one can see the importance of remote monitoring for the students, in a way that this teaching-learning modality contributed to the formation of students, benefiting both the monitor and the one who is a listener/learning partner, sharing knowledge, adding a new way of seeing subjects and practices, and, therefore, being fundamental in future moments, in which the student will become a better professional.
\end{abstract}

Keywords: Teaching; Learning; Health; Nursing; Covid-19.

\title{
Resumen
}

El monitorio es una de las actividades más importantes de las instituciones de educación superior que tiene una conjugación de educación, investigación y extensión, también hay monitoreo remoto, que tiene lo mismo objetivo y directorios que en el monitoreo presencial. En este sentido, el articulo tiene como objetivo describir la experiencia de estudiantes en el enfermaría graduación de la universidad CESMAC do Sertão. Es un estudio descriptivo, un relato de 
experiencias, cuya narrativa se basa en una observación sistematicé de la realidad. Se fue echo un elaboró informe de experiencias, con el fin de demostrar herramientas innovadoras para la enseñanza y el aprendizaje, describiendo las tecnologías de comunicación y informaciones que se fue utilizados. En esta perspectiva, ante el escenario de la COVID -19, se debieron adoptar nuevas medidas, la facultad adoptó el proyecto de monitoreo remoto que se distribuyó en disciplinas de determinadas épocas, posibilitando experiencias promoviendo la integración en los estudiantes, en el apoyo y desarrollo de la disciplina y aclaración de dados. A si, el programa de monitoreo remoto fue impartido por los estudiantes de la facultad bajo la guía de los respectivos profesores de las disciplinas. A partir de este estudio se destaca la importancia del monitoreo remoto para los estudiantes, por lo que esta modalidad del enseñanza y aprendizaje contribuyo a la formación de estés estudiantes, que se beneficiando tanto al que estaba en el rol de monitor como al que estaba escuchando como compañero de aprendizaje, compartiendo conocimiento, añadiéndole una nueva forma de ver los temas y las prácticas en los momentos futuros, en que el alumno le hará un mejor profesional.

Palabras clave: Enseñando; Aprendiendo; Salud; Enfermería; Covid-19.

\section{Introdução}

Segundo Haaget al. (2017), a monitoria é uma modalidade de ensino-aprendizagem que atende as necessidades de formação acadêmica e é destinada aos alunos regularmente matriculados em cursos de graduação. Essa prática contribui para a qualidade da formação dos estudantes por meio dos monitores, e, além disso, é uma atividade que faz parte de um processo educativo, tornando-se uma importante estratégia de trabalho no mundo acadêmico.

Diante disso, monitoria se caracteriza como uma das atividades de ensino, pesquisa e extensão mais importantes das instituições de nível superior. Além da riqueza para a formação acadêmica, a monitoria ajuda na criação de novos métodos de ensino, práticas lúdicas e pedagógicas. Nessa conformidade, Lopes (2010), ressalta que os discentes beneficiados pela monitoria possuem experiências escolares distintas, e que o programa de monitoria oferece a estes um meio de fixar e complementar o conteúdo já visto.

Nesse sentido, a monitoria acadêmica se baseia em atividades de ensino e aprendizagem estabelecidas pelo discente monitor como um meio de familiarizá-lo à prática da docência, o qual se assemelha a um estágio.

Dessa forma, sob a instrução de um docente, a monitoria auxilia o monitor a aprofundar e praticar os assuntos ministrados pelo professor em sala de aula e sanar as dificuldades e dúvidas que surgem no decorrer da disciplina, proporcionando momentos de debates e discussões sobre as temáticas abordadas (Antunes et al. 2018). Em vista disso, a monitoria passa a contribuir para a formação do aluno nas atividades acadêmicas, auxiliando os estudantes ao longo do processo, esclarecendo dúvidas e outras atividades definidas no plano de trabalho dos monitores, que são facilitadores deste processo.

De acordo com Frison (2016), a monitoria no nível superior tem sido importante na aprendizagem do alunado e utilizada constantemente nas Instituições, se destacando como uma estratégia que auxilia de modo direto no ensino dos alunos.

Seguindo esse contexto, há também a monitoria remota, que conjectura o mesmo objetivo e diretrizes da monitoria presencial, e que, por sua vez, terá o uso frequente das Tecnologias da Informação e Comunicação (TICs), na qual os monitores estarão participando efetivamente do processo de ensino-aprendizagem de discentes (Meirinhos, 2015, p.03).

Dito isto, sabe-se que as TICs estão cada vez mais presentes na vida do ser humano, e no meio educacional não tem sido diferente, pois as utilizações dos recursos das TICs estão crescendo na prática pedagógica, tornando-se um meio significativo no processo de ensino e aprendizagem, como o cinema, vídeo, rádio, TV, DVD, computadores, internet, máquina fotográfica, retroprojetor etc (Kwamme, 2018).

Com isso, Meirinhos (2015), ressalta que a interação e colaboração à distância são hoje uma realidade, que exigem novas abordagens pedagógicas para poder tirar proveito educativo da comunicação e acesso à informação virtualizada.

Em relação à monitoria remota, a realização de plantões on-line e interações por videoconferência são ferramentas encontradas na plataforma Microsoft Teams, que é uma das tecnologias que possibilitam uma comunicação fácil e direta dos estudantes com os monitores, além de conceder a dinamicidade entre professores e estudantes. 
À face do exposto, o presente artigo objetiva descrever a experiência vivenciada por acadêmicas do curso de graduação em enfermagem da Faculdade CESMAC do Sertão, durante as atividades desenvolvidas nas práticas de monitoria remota, relatando os resultados positivos, negativos, vantagens e dificuldades, como também acerca da importância desta experiência na vida acadêmica do aluno monitor, oferecendo assim subsídios para o exercício profissional.

\section{Referencial Teórico}

O referencial teórico da presente pesquisa foi estruturado em dois tópicos: A monitoria remota como modalidade de ensino e Tecnologias da Informação e Comunicação (TICs) no processo de ensino e aprendizagem.

\subsection{A Monitoria Remota Como Modalidade de Ensino}

A monitoria é uma modalidade de ensino que ajuda na aprendizagem e contribui na formação integrada ao discente nas atividades de ensino, sendo entendida como um instrumento para melhoria do ensino na graduação através do estabelecimento de novas práticas e experiências pedagógicas.

Sendo assim, tem como propósito apresentar para os discentes vinculados ao programa, a experiência na área da docência no qual o aluno monitor irá auxiliar outros alunos em determinada disciplina, de forma didática, comunicativa, ativa e dinâmica. Acerca disso, Soares (2008), em um estudo a respeito da importância da monitoria, demonstrou haver uma melhoria significativa no aproveitamento dos alunos que se dispuseram em participar das aulas de reforço (monitoria), em comparação aos que não o fizeram.

No presente relato de experiência, consideramos que foi primordial ao processo de formação pessoal, acadêmica e profissional dos monitores que viveram essa nova prática.

O monitor é o estudante que, interessado em desenvolver-se, aproxima-se de uma disciplina e junto com o professor realiza pequenas tarefas ou trabalhos que contribuem para o ensino, a pesquisa e extensão.

Nesse contexto, as atividades de monitoria dizem respeito às ações extraclasses a fim de auxiliar nas dificuldades ocorridas em sala de aula mediatizadas pelo monitor. Faria e Schneider (2006), ao se referirem ao trabalho de monitoria como um auxiliar da aprendizagem, destacam que a monitoria pode ser compreendida como uma atividade de suporte discente ao processo de ensino aprendizagem.

\subsection{Tecnologias da Informação e Comunicação (TICs) no Processo de Ensino e Aprendizagem}

Os benefícios que as tecnologias geram são inúmeros e se estendem em vários setores, de modo que as tecnologias da informação e comunicação vêm inovando a troca de informações entre pessoas, impactando diretamente no comportamento pessoal e profissional. Em relação à educação, as novas estratégias pedagógicas ligadas à tecnologia são de grande importância para o conhecimento.

Considerando as exigências de formação na área das Ciências da Saúde, diversos autores defendem o desenvolvimento de estratégias educativas inovadoras, baseadas no diálogo e na construção ativa de conhecimento pelos discentes (Mcallister, 2013; Rangel, 2011; Vyas, 2010).

Neste sentido, é evidente o atual sucesso da modalidade EaD, através das TICs, proporcionando uma evolução, tanto no processo de ensino quanto de aprendizagem. 


\section{Metodologia}

Trata-se de um estudo descritivo do tipo relato de experiência, cuja narrativa se baseia numa observação sistemática da realidade, correlacionando as descobertas com os embasamentos teóricos existentes (Steindorff, 2017, apud Dyniewicz, 2014).

Quanto a natureza, a pesquisa é de base qualitativa, onde está focada no desenvolvimento de pesquisas que buscam respostas e que viabilizem compreensão, descrição e interpretação dos elementos. Permitindo, assim, que o pesquisar tenha uma comunicação direta e participativa com o objetivo de estudo. Em linhas gerais, a natureza desse estudo apresenta narrativas, conceitos e experiências particulares dos participantes, possibilitando a compreensão da abrangente do fenômeno (Proetti, 2018).

O presente estudo aborda as vivências das acadêmicas do curso de graduação em enfermagem, sob orientação de professores das respectivas disciplinas no cumprimento de monitoria na modalidade remota, durante o primeiro semestre de 2020, mais precisamente entre os meses de maio a julho, com carga horária semanal de 12 horas.

A base metodológica utilizada foi composta pela Tecnologia da Informação e Comunicação (TIC), que segundo Lobo e Maia (2015), são definidos como um agrupamento de artifícios tecnológicos que, quando interligados entre si, possibilitam a automatização e/ou a comunicação nos processos existentes no ensino, pesquisa científica e etc. São, portanto, tecnologias empregadas para reunir, distribuir e compartilhar informações.

A efetividade desta ocorreu por meio de acesso on-line a plataforma digital Microsoft Teams, fazendo uso ainda do WhatsApp para interação/comunicação monitor-discente. Dentre os recursos utilizados para a realização das aulas, destacaramse as metodologias ativas que, conforme Morán (2015), nascem como ferramenta transformadora, a fim de gerar alunos proativos e criativos, com o auxílio de atividades em que tenham que tomar decisões e avaliar os resultados, além de conhecer novas possibilidades, sendo estes pontos importantes para progredir no tocante a reflexão, integração cognitiva e reelaboração de práticas inovadoras.

No contexto da monitoria remota, para o desenvolvimento e exposição de materiais dos conteúdos teóricos, adotaram-se como instrumento didático mapas mentais produzidos no Word e posteriormente convertidos em PDF, flash cards elaborados através do PowerPoint, jogos on-line como o Kahoot, gincanas com disciplinas integradas, confecção de cartazes educativos em aplicativos de aparelhos celulares e formulários no Google Forms.

Ao findar o processo de monitoria foi solicitado pela coordenação do curso um relatório final, para obtenção do certificado, e este era composto por: introdução destacando importância e conceito, objetivos, cronograma, estratégias didáticas, atividades desenvolvidas, evidências que compreendiam fotos/prints dos momentos reunidos com os discentes, dificuldades detectadas, considerações finais e referências.

\section{Resultados e Discussão}

Após a disseminação do Sars-CoV-2, causador da Covid-19, que veio a se tornar uma das pandemias mais mortais da humanidade, desafios foram encontrados para dar continuidade às atividades normais da população, a exemplo do processo de ensino e aprendizagem a nível superior que precisou se adequar a nova realidade vivenciada pelo Brasil e o mundo (Borba, 2020).

De acordo com esse contexto, evidencia-se a necessidade de métodos inovadores no processo pedagógico, pois, com a busca por formar e ferramentas inovadoras nessa ação, o processo de ensino e aprendizagem pôde se adequar a realidade vivida e até mesmo abranger o público beneficiado por esse processo (Muraro, 2019).

Nessa perspectiva, a Faculdade CESMAC do Sertão, situada na cidade de Palmeira dos Índios, no agreste de Alagoas, adotou o projeto de monitoria remota que foi distribuída em diversas disciplinas do curso de Bacharelado em Enfermagem. Partindo, então, da perspectiva de que a monitoria possibilita a experiência no meio acadêmico promovendo a integração entre 
os alunos, e a participação nas funções de organização e no apoio ao desenvolvimento da disciplina e no esclarecimento de dúvidas.

Desse modo, o programa de monitoria remota foi efetivado pelos discentes da Faculdade sob a orientação dos respectivos professores das disciplinas que estavam no programa. Com duração de dois (02) meses, as disciplinas que tiveram o auxílio da monitoria foram: Atenção Integral a Saúde da Mulher I e II, Organização Morfológica e Funcional I e II e Bases Celulares e Moleculares.

Ao professor-orientador, foi atribuída a função de orientar o processo de ensino-aprendizagem dos alunos, que foram realizados pelo monitor, assegurando o cumprimento dos objetivos do Programa de Monitoria, cabendo ao professororientador propor atividades, sugerindo, quando necessário, fontes adicionais de informações educacionais.

Ministrada em diversos períodos do Curso de Graduação em Enfermagem, a monitoria remota teve carga horária total de 12 horas semanais. A abordagem adotada nas diversas disciplinas foi à metodologia ativa que considera o educando como sujeito elaborador do conhecimento, inserido em um contexto de conhecimento compartilhado entre o monitor-discente.

Com isso, segundo Moran (2018), as metodologias ativas de ensino e de aprendizagem estão sendo gradativamente implantadas em alguns cursos do ensino superior, e elas constituem alternativas pedagógicas que colocam o foco do processo de ensino-aprendizagem no aluno, envolvendo-o na aprendizagem por descoberta, por investigação ou resolução de problemas.

A educação elaborada a partir dos saberes prévios do monitor possibilita a constituição desse ensino-aprendizagem de forma significativa, por meio do comportamento crítico-reflexivo, de modo que o conhecimento terá aplicabilidade e relevância na vida profissional de cada um.

Dessa forma, para a prática da monitoria remota, inicialmente foram criadas salas virtuais no perfil da plataforma Microsoft Teams para todas as turmas participantes da monitoria como mostra na Figura 1, e esse seria o principal meio para a realização dos encontros semanais.

A plataforma funciona como uma central de informações, discussões e colaborações, com o intuito de auxiliar o processo de aprendizagem na faculdade e ampliar o vínculo dos estudantes na postagem de tarefas e discussão das temáticas apresentadas.

Figura 1 - Salas virtuais criadas na plataforma Microsoft Teams.

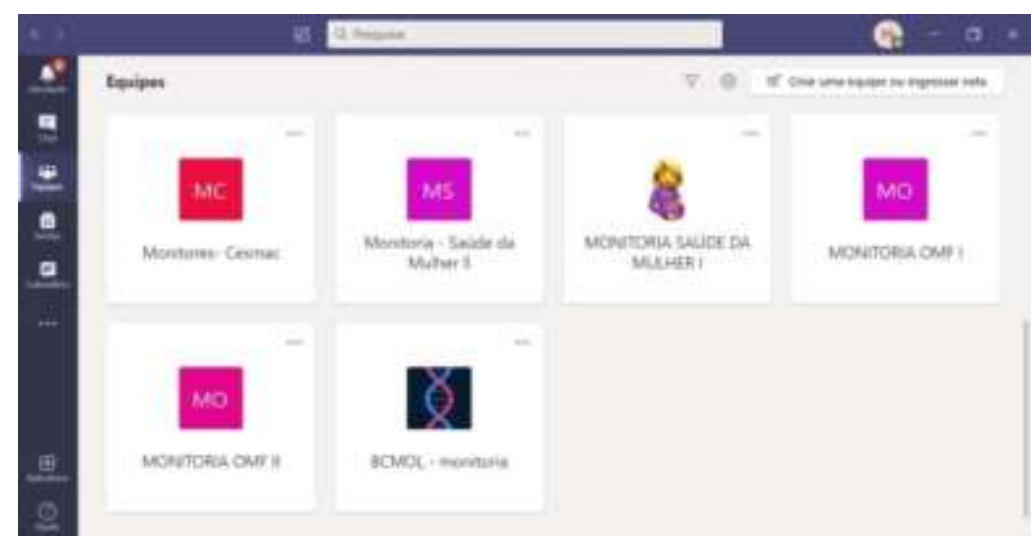

Fonte: Autores (2020).

Apesar de a plataforma Microsoft Teams ser a principal estratégia para a realização da monitoria, grupos no Whatsaap também foram criados para garantir uma comunicação mais efetiva e de forma mais rápida, visto que as monitorias aconteciam três (03) vezes por semana para cumprimento de carga horária estabelecida pela instituição. 
Após isso, iniciaram-se os encontros com essas turmas, sendo às temáticas abordadas de forma lúdica sempre com auxílio de recursos que facilitassem uma melhor adesão do conteúdo, como, por exemplo, slides, mapas mentais, flash cards, jogos on-line, como o Kahoot, gincanas com disciplinas integradas, confecção de cartazes educativos e formulários no Google Forms.

Na figura a seguir trouxemos um modelo de flash card que foi utilizado na monitoria, no qual foi criado uma espécie de "cartão rápido" que possui como objetivo principal facilitar a revisão dos conteúdos.

Figura 2 - Flash card da disciplina Atenção Integral a Saúde da Mulher II

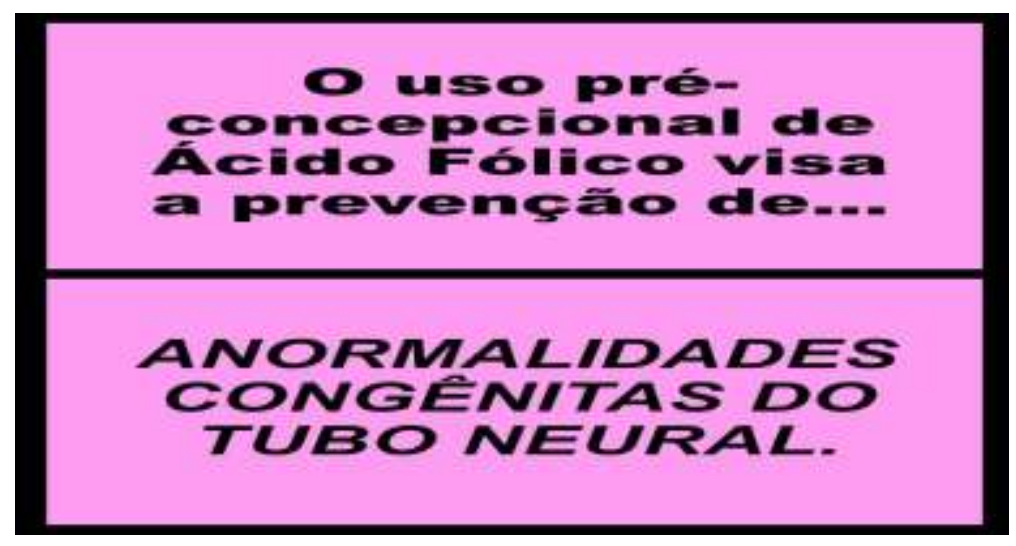

Fonte: Autores (2020).

Ainda seguindo com o uso do flash card, a Figura 3 traz mais um modelo que foi associado com a prática de mapas mentais, e o uso dessas duas ferramentas só vem a contribuir, facilitando o aprendizado de conteúdos mais extensos.

Figura 3 - Flash card da disciplina Atenção Integral a Saúde da Mulher I.

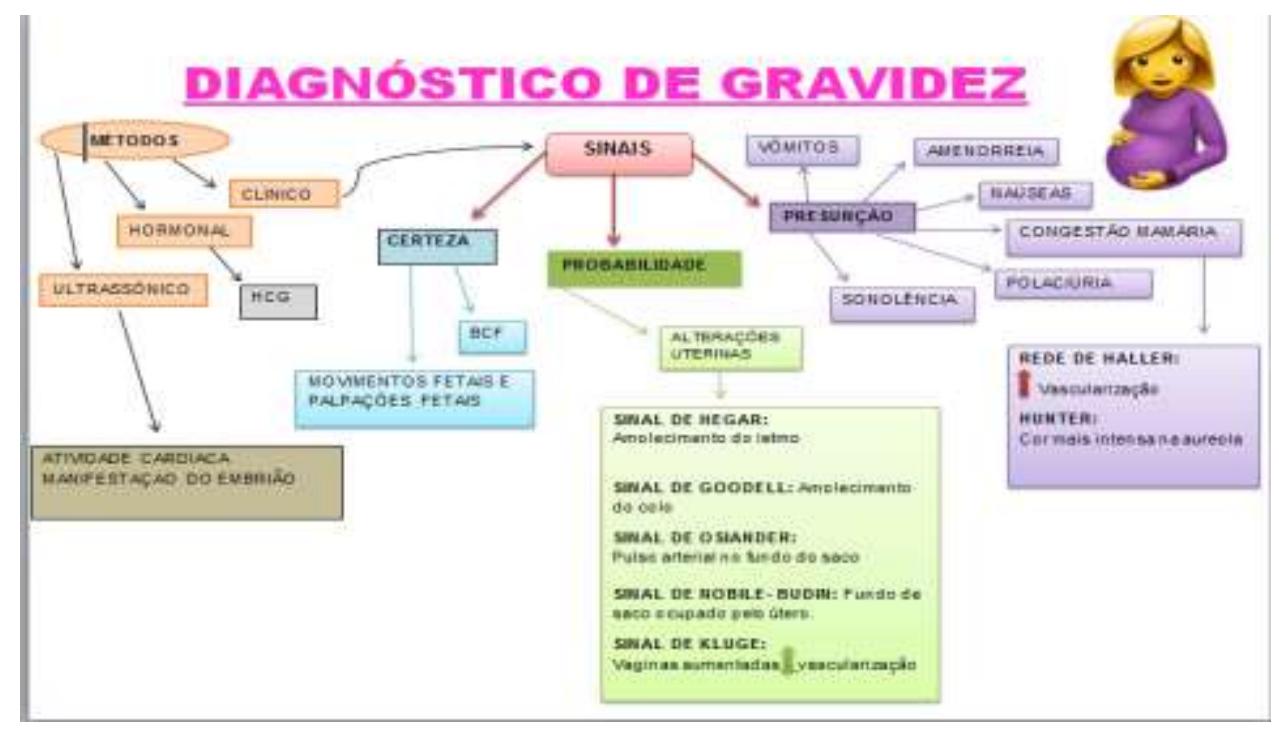

Fonte: Autores (2020).

Com o ensino de forma remota outra ferramenta que também ganhou espaço foram os jogos educativos, e como exemplos temos a Figura 4, sendo um jogo realizado em equipe de perguntas e resposta, bastante utilizado para revisar conteúdos dias antes das avaliações obrigatórias das disciplinas. 
Figura 4 - jogo online no Kahoot na disciplina de Organização Morfológica e Funcional I.

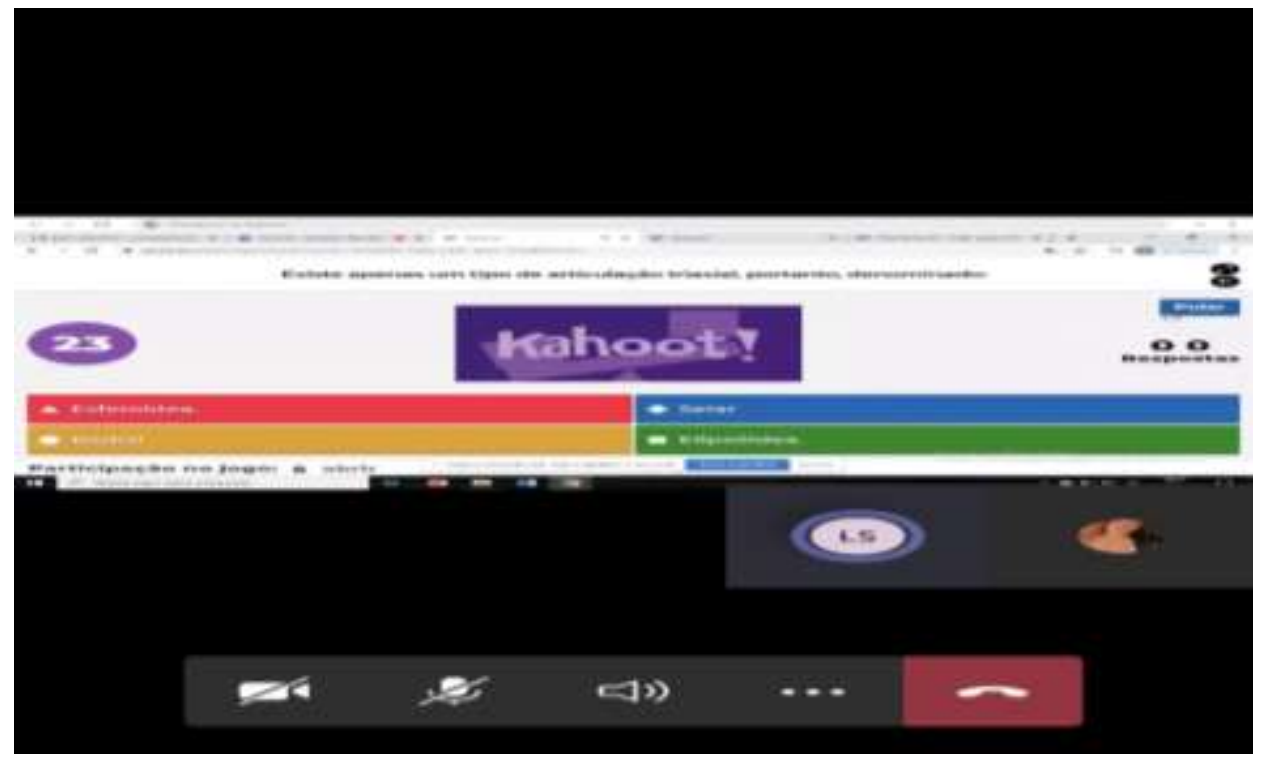

Fonte: Autores (2020).

A plataforma Google por si só é bastante utilizada por várias pessoas, mas após a necessidade de inovação essa plataforma passou a ser mais explorada e nessa busca o Google Forms também ganhou o seu espaço, e como é mostrada na Figura 5, essa ferramenta permite a elaboração de questionários e vem sendo bastante utilizada para aplicação de testes e avaliações pelas instituições de ensino.

Figura 5 - questionário elaborado no Google Forms na disciplina de Atenção Integral a Saúde da Mulher II.

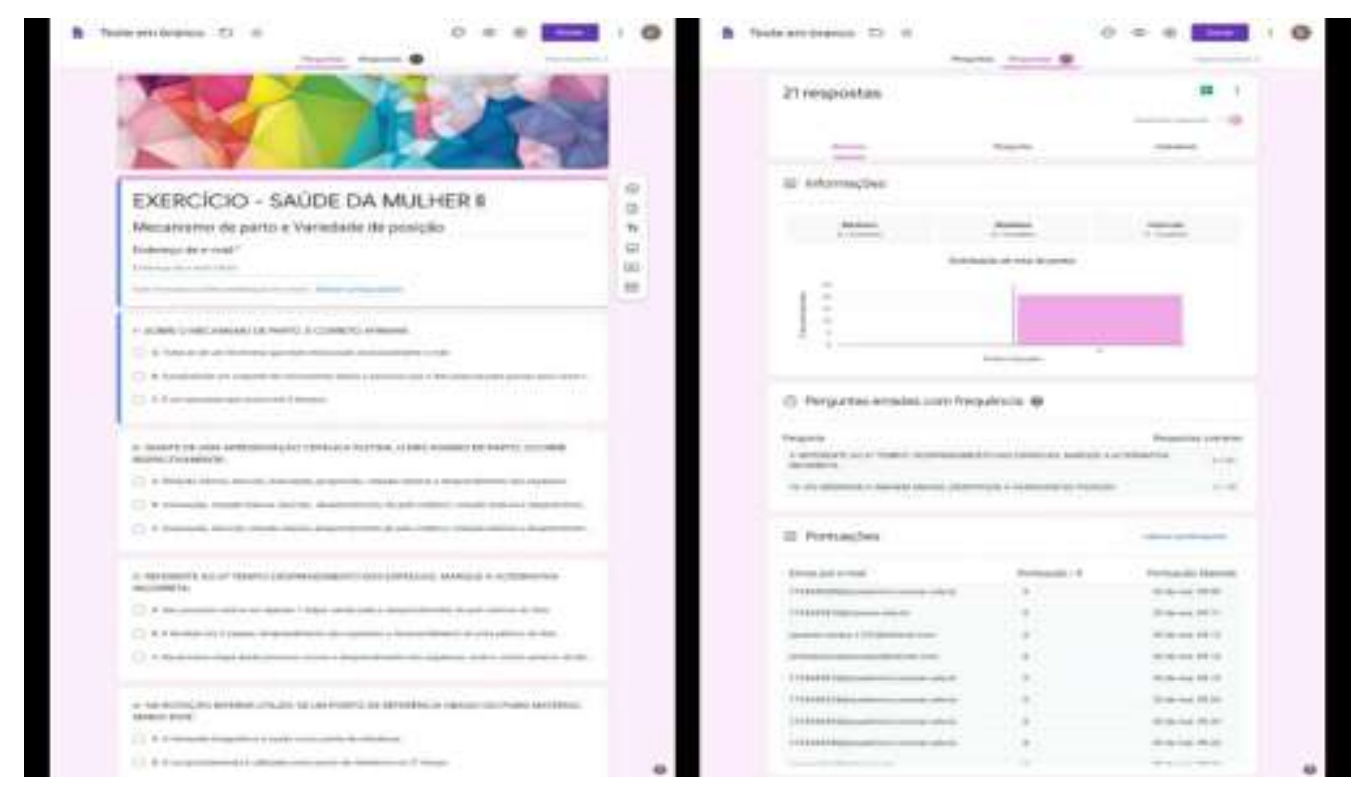

Fonte: Autores (2020).

Na Figura 6 o cartaz educativo é ilustrado na sua forma digital, com um texto curto e objetivo e com a utilização de uma linguagem de fácil entendimento, pois o seu intuito é fornecer suporte ao estudante com dicas relevantes para produção de trabalhos acadêmicos. 
Figura 6 - Cartaz educativo.

\section{DICAS PARA CONSTRUÇÃO DE TRABALLIOS NO PONEGTPOINT}

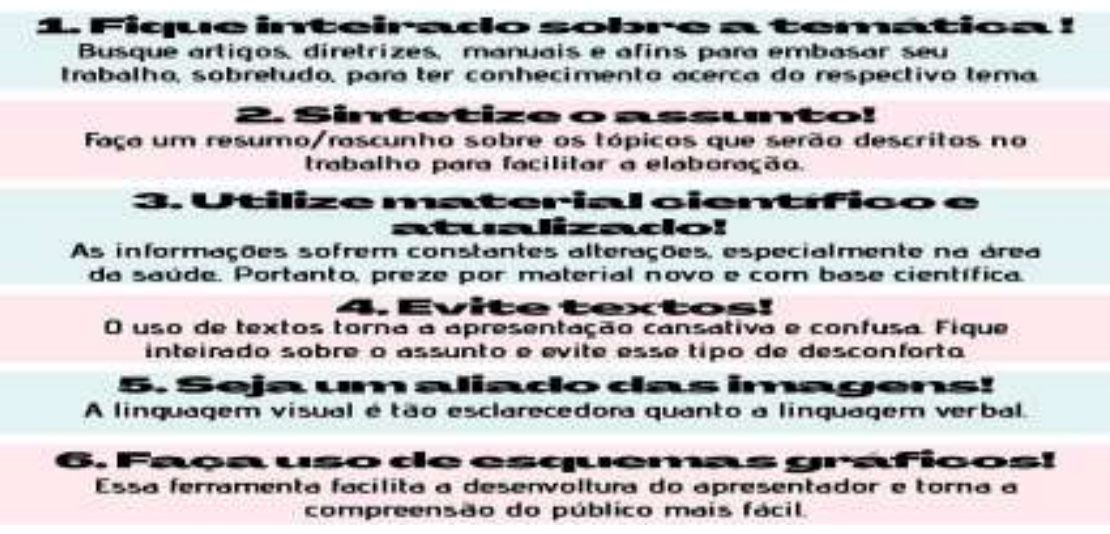

Fonte: Autores (2020).

No total foram realizados 45 encontros de monitora remota. Um total de cinco turmas foi contemplado com esse programa. Para execução de cada monitoria remota todo material produzido era analisado com antecedência pelo professor da disciplina, a fim de avaliar e corrigir o que fosse necessário.

No final da monitoria remota os alunos também foram questionados sobre sua opinião acerca do oferecimento da monitoria na sua forma virtual que está representado no quadro 1, e dessa forma, puderam destacar a relevância e importância de estarem sempre revisando os conteúdos ministrados pelo professor. Com isso, foi possível identificar pontos positivos e pontos negativos acerca da utilização do ambiente virtual como ferramenta para a sua formação.

Quadro 1- avaliação da monitoria remota pelos alunos participantes.

\begin{tabular}{|l|l|}
\hline PONTOS POSITIVOS & PONTOS NEGATIVOS \\
\hline Possibilidade de correção de trabalhos & Os horários estabelecidos \\
Várias metodologias utilizadas & Sobrecarga de todas as disciplinas \\
Melhor fixação dos conteúdos & Dificuldade de acesso à internet \\
Interação entre o aluno-monitor & Carga horária extensa \\
Disponibilidade e receptividade além dos horários & \\
da monitoria. & \\
\hline
\end{tabular}

Fonte: Autores (2020).

A discussão sobre esses pontos, tanto os positivos quanto os negativos foram de suma relevância, mostrando o quanto foi eficaz a utilização dessas metodologias ativas, pois, apesar das dificuldades encontradas, a forma da abordagem metodológica foi altamente aprovada por todos os alunos que participaram, e ter esta proximidade com a tecnologia nos possibilitou melhores experiências no ensino-aprendizagem. 


\section{Considerações Finais}

O presente relato de experiência demonstra a importância da monitoria de forma remota para os alunos de diversos cursos, a exemplo dos alunos do curso de graduação em Enfermagem. Tal modalidade de ensino-aprendizagem contribuiu na formação de estudantes, beneficiando tanto os estudantes-monitores quanto os estudantes-ouvintes, estabelecendo uma parceria de aprendizado em que ambos compartilharam conhecimentos, auxiliando numa nova forma de enxergar assuntos e práticas fundamentais para a formação profissional dos discentes.

Torna-se relevante salientar que, mediante a decisão institucional da Faculdade CESMAC do Sertão, a proposta da monitoria remota se tornou uma ferramenta indispensável em um período difícil e totalmente novo devido a pandemia. A monitoria remota, portanto, se configurou como um método inovador para transformar o ensino tradicional em uma nova forma de reagir à situação atua, na qual as aulas presenciais foram paralisadas e o espaço entre professor e aluno ficou distante fisicamente. Assim, a monitoria remota interviu e estreitou laços, aproximando alunos e agregando conhecimento a todos que participaram do programa.

Vale ressaltar o total apoio dos discentes ouvintes, facilitando o desenvolvimento do programa. Em dois meses foram realizadas atividades remotas de cunho não obrigatório, mas que elevou o nível de conhecimento. Além disso, a Faculdade CESMAC do Sertão se fez presente e esteve em total apoio aos seus estudantes, agindo com plena comunicação e cuidado para efetivar a melhor monitoria remota possível. Por fim, os docentes estabeleceram um grande contato e aproximação com seus monitores, gerando resultados positivos a esse novo método de ensino-aprendizagem.

O aluno monitor, por sua vez, teve de aprender com amigos e parceiros que, levando em consideração as relações de proximidade entre ambos, podendo efetivar um melhor aprendizado. Assim sendo, a monitoria remota beneficiou a todos e foi validada da mesma forma que a monitoria presencial, pois sua relevância e a atenção prestada aos alunos foram às mesmas desenvolvidas presencialmente.

Com isso, a monitoria remota fortaleceu deias e teorias, na qual demonstrou que não é necessário estar presente fisicamente para existir uma troca de conhecimento. Em suma, esta monitoria ensinou ainda aos monitores acerca da responsabilidade e comprometimento, tornando-os mais maduros e experientes.

Por tudo que foi mencionado, essa modalidade de ensino e aprendizagem deixará frutos que servirão para as gerações futuras, pois, o aprimoramento dessa prática juntamente com o uso das tecnologias inovadoras de comunicação e novas descobertas que irão advir, são sugestões e vantagens que irão se agregar na vida do estudante somando pontos positivos.

\section{Referências}

Antunes, S. S; et al. (2018). A importância do monitor para o processo de formação acadêmica, otimizando o aprendizado. Revista Cintedi, Campina Grande.

Borba, P. L. O; et al. (2020). Desafios 'práticos e reflexivos' para os cursos de graduação em terapia ocupacional em tempos de pandemia. Cadernos Brasileiros de Terapia Ocupacional/Brazilian Journal of Occupational Therapy, Scielo Preprint.

Faria, J. P. (2003). A monitoria como prática colaborativa na universidade. Dissertação de Mestrado. Programa de Pós-Graduação em Linguística Aplicada e Estudos da Linguagem. Pontifícia Universidade Católica de São Paulo.

Frison, L. (2016). Monitoria: uma modalidade de ensino que potencializa a aprendizagem colaborativa e autorregulada. Pro-Posições. v. 27, n. 1, p. 133-153, jan. /abr.

Haag, G. S; et al. (2017). Contribuições da monitoria no processo ensino-aprendizagem em enfermagem. Rev. brasileira de enfermagem, Brasília- DF, v. 61, n. 2, p. 215-220, Abr.

Kwamme, C. M. L. (2018). As contribuições da monitoria: monitor em formação acadêmica. Anais do $10^{\circ}$ Salão Internacional de Ensino Pesquisa e Extensão-SIEPE; Universidade Federal do Pampa, Santana do Livramento.

Lobo, A. S. M. \& Maia, L. C. G. (2015). O uso das TICs como ferramenta de ensino-aprendizagem no Ensino Superior. Caderno de Geografia, v. 25, n. 44, p. $16-26$.

Lopes, C. S. \& Espig, M. J. (2010). A importância da atividade do monitor na universidade: um estudo de caso na teoria da história. In: Congresso de Iniciação Científica, 19.; Encontro de pós-graduação, mostra científica, Pelotas: UFPel. 
Mcallister, M. et al. (2013). Snapshots of simulation: creative strategies used by Australian educators to enhance simulation learning experiences for nursing students. Nurse Education in Practice, Edinburgh, v. 13, n. 6, p. 567-572.

Meirinhos, M. (2015). Os desafios educativos da geração Net. Revista de Estudios e Investigación em Psicología y Educación (13), 125-129.doi: 10.17979/reipe.2015.0.13.453

Moran, J. (2018) O papel das metodologias na transformação da Escola. http://www2.eca.usp.br/moran/wpcontent/uploads/2013/12/Papel_metodologias_Moran.pdf

Muraro, P. F. L. (2019). Inovação em ensino e aprendizado de histologia: atlas direcionado a acadêmicos da área da saúde. Anais do 10 ${ }^{\circ}$ Salão Internacional de Ensino, Pesquisa e Extensão-SIEPE.

Proetti, S. (2018) As pesquisas qualitativa e quantitativa como métodos de investigação científica: Um estudo comparativo e objetivo. Revista Lumen-ISSN: 2447-8717, v. 2, n. 4.

Rangel, E. (2011). Avaliação, por graduandos de enfermagem, de ambiente virtual de aprendizagem para ensino de fisiologia endócrina. Acta Paulista de Enfermagem, São Paulo, v. 24, n. 3, p. 327-333.

Steindorff, G. (2017). Monitoria acadêmica no componente curricular de Semiotécnica em Enfermagem: Relato de experiência. Anais do Salão Internacional de Ensino, Pesquisa e Extensão, v. 8, n. 1.

Vyas, R. (2010). Clinical training at remote sites using mobile technology: an India-USA partnership. Distance Education, London, v. 31 , n. 2, p. 211-216. 